\title{
SNNRice6mA: A Deep Learning Method for Predicting DNA N6-Methyladenine Sites in Rice Genome
}

\author{
Haitao $Y u^{1}$ and Zhiming Dai ${ }^{1,2 *}$ \\ ${ }^{1}$ School of Data and Computer Science, Sun Yat-Sen University, Guangzhou, China, ${ }^{2}$ Guangdong Province Key Laboratory \\ of Big Data Analysis and Processing, Sun Yat-Sen University, Guangzhou, China
}

DNA N6-methyladenine (6mA) is an important epigenetic modification, which is involved in many biology regulation processes. An accurate and reliable method for $6 \mathrm{~mA}$ identification can help us gain a better insight into the regulatory mechanism of the modification. Although many experimental techniques have been proposed to identify $6 \mathrm{~mA}$ sites genome-wide, these techniques are time consuming and laborious. Recently,

OPEN ACCESS

Edited by: Harinder Singh,

J. Craig Venter Institute,

United States

Reviewed by:

Quan Zou,

University of Electronic Science and Technology of China, China

Kumardeep Chaudhary,

Icahn School of Medicine at

Mount Sinai, United States

${ }^{*}$ Correspondence Zhiming Dai

daizhim@mail.sysu.edu.cn

Specialty section: This article was submitted to Bioinformatics and Computational Biology, a section of the journal

Frontiers in Genetics

Received: 15 July 2019 Accepted: 04 October 2019 Published: 11 October 2019

Citation:

Yu H and Dai Z (2019) SNNRice6mA:

A Deep Learning Method for

Predicting DNA N6-Methyladenine

Sites in Rice Genome.

Front. Genet. 10:1071.

doi: 10.3389/fgene.2019.01071 several machine learning methods have been developed to identify $6 \mathrm{~mA}$ sites genomewide. However, there is room for the improvement on their performance for predicting $6 m A$ sites in rice genome. In this paper, we developed a simple and lightweight deep learning model to identify DNA 6mA sites in rice genome. Our model needs no prior knowledge of $6 \mathrm{~mA}$ or manually crafted sequence feature. We built our model based on two rice $6 \mathrm{~mA}$ benchmark datasets. Our method got an average prediction accuracy of $\sim 93 \%$ and $\sim 92 \%$ on the two datasets we used. We compared our method with existing $6 \mathrm{~mA}$ prediction tools. The comparison results show that our model outperforms the state-of-the-art methods.

Keywords: epigenetics, deep learning, DNA sequence, rice, bioinformatics

\section{INTRODUCTION}

DNA N6-methyladenine $(6 \mathrm{~mA})$ is one important DNA epigenetic modification, which has been found in bacteria, eukaryote, and archaea (O'brown and Greer, 2016). It was reported that $6 \mathrm{~mA}$ is involved in many biological processes. For example, in bacteria, $6 \mathrm{~mA}$ plays roles in DNA replication (Campbell and Kleckner, 1990), DNA repair (Au et al., 1992), transcription (Robbins-Manke et al., 2005), and gene expression regulation (Low et al., 2001). But for eukaryote, the study of DNA 6mA"'” is still insufficient (Koziol et al., 2016). Studying the distribution of DNA 6mA can provide a deeper understanding of the epigenetic modification process.

Recently, the development of experimental techniques contributes to studying DNA 6mA modification. Pormraning et al. developed a protocol using bisulfite sequencing and methyl-DNA immunoprecipitation technique to analyze genome-wide DNA methylation in eukaryotes (Pomraning et al., 2009). Krais et al. reported a fast and sensitive method for the quantification of global adenine methylation in DNA, using laser-induced fluorescence and capillary electrophoresis (Krais et al., 2010). Flusberg et al. applied single-molecule, real-time sequencing technique to detect DNA methyladenine directly (Flusberg et al., 2010). Greer et al. used ultra-high performance liquid chromatography coupled 
with mass spectrometry technique to access DNA 6mA levels in Caenorhabditis elegans (Greer et al., 2015).

Due to the large cost of experiment-based approach, researchers have used machine learning technology to identify and predict DNA $6 \mathrm{~mA}$ sites. Feng et al. proposed a SVMbased tool (called iDNA6mA-PseKNC) to predict $6 \mathrm{~mA}$ sites in Mus musculus genome (Feng et al., 2019). Feng et al. validated iDNA6mA-PseKNC on the M. musculus dataset and other $6 \mathrm{~mA}$ datasets of eight microbe species. It was reported that iDNA6mA-PseKNC achieved very high prediction accuracy on all the nine datasets, revealing that this method is reliable and can identify genome-wide $6 \mathrm{~mA}$ sites in many species. Recently, Chen et al. provided a benchmark $6 \mathrm{~mA}$ dataset containing 880 $6 \mathrm{~mA}$ sites and 880 non- $6 \mathrm{~mA}$ sites in rice genome (Chen et al., 2019), which was denoted as $6 \mathrm{~mA}$-rice-Chen in this study. By using many manually crafted DNA sequence features, they built a support vector machine (SVM) based tool (called i6mA-Pred) to identify $6 \mathrm{~mA}$ sites in rice genome. It was reported that $\mathrm{i} 6 \mathrm{~mA}-$ Pred got an accuracy of $\sim 83 \%$ on the rice genome dataset. Pian et al. proposed a tool, called MM-6mAPred, based on the markov model for $6 \mathrm{~mA}$ sites prediction (Pian et al., 2019). Pian et al. built and evaluated their MM-6mAPred based on the 6mA-riceChen benchmark dataset. It was reported that MM-6mAPred outperformed i6mA-Pred in prediction of $6 \mathrm{~mA}$ sites. Tahir et al. proposed another computational tool, called iDNA6mA, for $6 \mathrm{~mA}$ identification in rice genome (Tahir et al., 2019). Tahir et al. trained and evaluated their iDNA6mA on the $6 \mathrm{~mA}$-riceChen dataset, and they found that iDNA6mA outperformed i6mA-Pred in prediction performance. Basith et al. proposed a tool, named SDM6A, for predicting $6 \mathrm{~mA}$ sites in the rice genome (Basith et al., 2019). SDM6A is an ensemble approach using several features encoding methods and machine learning classifiers. Basith et al. trained and evaluated their SDM6A based on the $6 \mathrm{~mA}$-rice-Chen benchmark dataset, and they found that SDM6A outperformed i6mA-Pred and iDNA6mA on the $6 \mathrm{~mA}$-rice-Chen benchmark dataset. Very recently, Lv et al. proposed a computational tool, iDNA6mA-rice, for prediction of $6 \mathrm{~mA}$ sites in rice genome ( $\mathrm{Lv}$ et al., 2019). Lv et al. proposed another $6 \mathrm{~mA}$ benchmark dataset for rice genome, and we denoted such dataset as $6 \mathrm{~mA}$-rice-Lv. The $6 \mathrm{~mA}$-rice-Lv contains $154,0006 \mathrm{~mA}$ sites-contained sequences as the positive samples and the same number of negative samples. Lv et al. trained and evaluated iDNA6mA-rice on $6 \mathrm{~mA}$-rice- $\mathrm{Lv}$ dataset by five-fold cross-validation, and they found that iDNA6mA-rice achieved good prediction performance. For the purpose of the comparison with i6mA-Pred, Lv et al. also trained and evaluated iDNA6mArice on the $6 \mathrm{~mA}$-rice-Chen dataset and found that iDNA6mArice outperformed i6mA-Pred on the $6 \mathrm{~mA}$-rice-Chen dataset.

Previous studies have shown that deep learning is a powerful technique for sequences analysis and classification in bioinformatics (Zhang et al., 2018; Zou et al., 2019). In this paper, we proposed a simple, lightweight, and high-performance method to improve prediction accuracy of DNA $6 \mathrm{~mA}$ sites in rice genome (called SNNRice6mA). SNNRice6mA is based on convolutional neural network architecture. It needs no manually crafted sequence feature and can learn high level abstract features, compared with traditional machine learning based methods. SNNRice6mA got an accuracy of $\sim 93 \%$ and $\sim 92 \%$ on the $6 \mathrm{~mA}$-rice-Chen and $6 \mathrm{~mA}$-rice-Lv datasets, respectively. SNNRice6mA performed better than previous methods in prediction of DNA $6 \mathrm{~mA}$ sites in rice genome.

\section{METHODS}

\section{Dataset}

In this study, we considered two $6 \mathrm{~mA}$ benchmark datasets for rice genome. The first dataset is the $6 \mathrm{~mA}$-rice-Chen dataset (Chen et al., 2019), which was widely used by previous studies (Basith et al., 2019; Chen et al., 2019; Pian et al., 2019; Tahir et al., 2019). The $6 \mathrm{~mA}$-rice-Chen dataset contains 880 positive samples and 880 negative samples. The second dataset we used is the $6 \mathrm{~mA}$-rice- $\mathrm{Lv}$ dataset ( $\mathrm{Lv}$ et al., 2019). The $6 \mathrm{~mA}$-rice- $\mathrm{Lv}$ dataset contains 154,000 positive samples and 154,000 negative samples. DNA sequences in both positive samples and negative samples are $41 \mathrm{bp}$ long. For each positive sequence, its center is the $6 \mathrm{~mA}$ modification site. For each negative sequence, its center contains no $6 \mathrm{~mA}$ modification site. By using these two widely used datasets, we can compare our method with previous methods fairly.

\section{The SNNRice6mA Method \\ Data Representation}

The samples in our dataset are DNA sequences, expressed in a string form. For example, a sample is like "GTATAT... GCCTAA." Before feeding the sequences to the model, we should first convert the sequence into encoding tensor.

Previous methods, including i6mA-Pred, iDNA6mAPseKNC, SDM6A, and iDNA6mA-rice, used manually crafted sequences features to represent DNA sample sequences, such as nucleotide chemical properties and nucleotide frequency (Basith et al., 2019; Feng et al., 2019; Lv et al., 2019). Manually crafted sequences features require a large amount of prior knowledge of DNA $6 \mathrm{~mA}$ modification and may be unsuitable for large data size.

Instead of using manually crafted DNA sequences features, we used the one-hot encoding method. A, T, C, and G are encoded as $(1,0,0,0),(0,1,0,0),(0,0,1,0)$, and $(0,0,0,1)$, respectively. Each sample sequence in our dataset is $41 \mathrm{bp}$ long. After one-hot encoding, each sequence is converted to a matrix, which has 41 columns and 4 rows. Each column of the matrix represents a specific DNA base of the sample sequence. In brief, the information fed to our model is only the base composition of a sample sequence, without any manually crafted DNA sequences feature.

\section{Model Details}

We built a deep learning method, called SNNRice6mA, based on the rice genome benchmark datasets. The architecture of our method is a typical convolutional neural network. SNNRice6mA contains eight components, constructed in a stacked way. The input vector of SNNRice $6 \mathrm{~mA}$ is a one-hot encoding DNA sequence. The first component of SNNRice6mA is a one-dimensional convolution layer, which is abbreviated as Conv. The layer Conv has 16 filters, whose lengths are all 4. 
Every filter in the layer Conv functions like a sequence motif recognizer of $6 \mathrm{~mA}$ modification sites in rice genome. For each input vector, each filter searches sequence patterns that can discriminate true $6 \mathrm{~mA}$ containing sequences from pseudo ones. To avoid overfitting, we used the L2 regularization method for the filter weights and bias in Conv layer. And we set all regularization rates as 0.0001 . The activation function used in Conv layer is the exponential linear unit (ELU) activation function. The second component of SNNRice $6 \mathrm{~mA}$ is a group normalization layer (GN), which aims to reduce the correlation of the results produced by each filter in Conv layer. Group normalization is suitable for the small size of the training batch (Wu and He, 2018). The GN divides the outputs of Conv layer into some groups and carries out the normalization in each group, respectively. We set the number of groups as four in GN. The third component of SNNRice $6 \mathrm{~mA}$ is a one-dimensional max pooling layer, reducing the redundancy of the features that the previous layer outputs. We set the size of the max pooling windows as 4 , which is the same as the size of the filter in convolution layer. We used the dropout layer after the pooling layer. The dropout layer acts like a regularization function to prevent overfitting of the model. In each training iteration, the dropout layer randomly shuts down some hidden neurons units by setting the outputs of these units to zero. So, after the dropout process, some intermediate features are discarded, which prevents overfitting and can improve the reliability and robustness of the model. We set the dropout rate as 0.25. After dropout layer, we used a flatten function to integrate the intermediate features, which are fed to the fully connected (FC) layer. The FC layer has 32 hidden units. To avoid overfitting, we used the $\mathrm{L} 2$ regularization method for the weights and bias. And we set all regularization rates as 0.0001 . The activation function used in FC layer is ELU activation function. The output of FC layer is fed to the last component, sigmoid function. The sigmoid function outputs a float value between 0 and 1 , which is considered as the probability of the input DNA sequence containing $6 \mathrm{~mA}$ modification site. If the probability is larger than 0.5 , the model will classify the input DNA sequence as the positive sample, which means the center of input DNA sequence is the $6 \mathrm{~mA}$ site. If the probability is smaller than 0.5 , the discrimination is the opposite.

We used the optimizer, stochastic gradient descent (SGD) with momentum, and the binary cross-entropy loss function. We set the learning rate as 0.005 and the momentum rate as 0.95 in SGD optimizer. We set the maximum training epoch as 100 and the batch size of training as 32 . We used early stopping technique in the training process. The early stopping means that the training process will stop when the prediction accuracy stops improving on the validation set. We set patience of early stopping as 30 epochs, which means that the training is stopped when the prediction accuracy on validation set does not improve after 30 training epochs. We also used the model checkpoint technique, which saves the model which has the highest prediction accuracy on the validation set. During the training process, we reduced the learning rate when the value of loss function on validation set no longer decreased. We set the reduced factor as 0.1 and the patience as 20 epochs, which means that the learning rate is reduced when the value of loss function on validation set does not improve after 20 training epochs.

We implemented our method based on Keras 2.2.4. We used the default values of hyper-parameters in Keras, except those that have been mentioned in this paper (see the full list in Table S1).

\section{Performance Metrics}

To be consistent with previous studies (Lv et al., 2019; Pian et al., 2019), we used the standard 10-fold cross-validation method to evaluate our method on the $6 \mathrm{~mA}$-rice-Chen dataset and used the standard 5-fold cross-validation method to evaluate our method on the $6 \mathrm{~mA}$-rice-Lv dataset. For example, in 10-fold-crossvalidation, we randomly partitioned the rice genome benchmark dataset into 10 folds with equal size. In each cross-validation iteration, we used eight folds for training, one fold for validating, and the remaining one fold for testing. In each iteration, we saved the specific model with highest accuracy on the validation fold and evaluated the performance of this model on testing fold. The cross-validation iteration was executed 10 times, and the average predicted accuracy of 10 iterations was calculated. Our source codes are available on https://github.com/yuht4/SNNRice6mA.

For the evaluation metrics, we used the same metrics as those in a previous study (Chen et al., 2019). Totally, five metrics have been used, including accuracy, sensitivity, specificity, Matthews correlation coefficient (MCC), and area under the curve (AUC).

The metric accuracy means the ratio of correct predictions on the testing data. The accuracy is defined as below:

$$
\text { accuracy }=\frac{\mathrm{TP}+\mathrm{TN}}{\mathrm{TP}+\mathrm{TN}+\mathrm{FP}+\mathrm{FN}}
$$

True positive (TP) is the number of predictions that classify the TP samples correctly. True negative (TN) is the number of predictions that classify the TN samples correctly. False positive (FP) is the number of predictions that misclassify the negative samples as the positive ones. False negative (FN) is the number of predictions that misclassify the positive samples as the negative ones. The positive means the samples containing the $6 \mathrm{~mA}$ sites, and vice versa.

The metric sensitivity is the ratio of correctly identified positive samples in all actual positive data. The sensitivity is defined as below:

$$
\text { sensitivity }=\frac{\mathrm{TP}}{\mathrm{TP}+\mathrm{FN}}
$$

The metric specificity is the ratio of correctly identified negative samples in all actual negative data. The specificity is defined as below:

$$
\text { specificity }=\frac{\mathrm{TN}}{\mathrm{TN}+\mathrm{FP}}
$$

MCC is a measure of the quality of binary classification model (Matthews, 1975). MCC takes TP, TN, FP, and FN into 
account. MCC is generally regarded as a balanced measure that can be used even if the samples are unbalanced in two classes (Boughorbel et al., 2017).

The MCC measures the correlation between the real and predicted binary classifications. MCC is a coefficient value between -1 and +1 . A value of +1 represents a perfect binary classification model, 0 means the same as random prediction, and -1 indicates total disagreement between predicted labels and real labels.

MCC is defined as below:

$$
\mathrm{MCC}=\frac{\mathrm{TP} \times \mathrm{TN}-\mathrm{FP} \times \mathrm{FN}}{\sqrt{(\mathrm{TP}+\mathrm{FP})(\mathrm{TP}+\mathrm{FN})(\mathrm{TN}+\mathrm{FP})(\mathrm{TN}+\mathrm{FN})}}
$$

AUC is the area value under the receiver operating characteristic curve. AUC is also an important indicator to measure the performance of a binary classification model. The larger the AUC value, the better the performance of model. AUC is a float value between 0 and 1; 1 means the model is perfect in prediction, while 0.5 means the model is similar with random predictions.

\section{RESULTS}

We evaluated the performance of our method SNNRice6mA on two DNA $6 \mathrm{~mA}$ benchmark datasets (i.e., $6 \mathrm{~mA}$-rice-Chen and $6 \mathrm{~mA}-$ rice-Lv) for rice genome. SNNRice6mA showed good performance on these two datasets in terms of different evaluation metrics (Figures 1, S1, S2 and Tables S2, S3). We compared SNNRice6mA with state-of-the-art tools. Results showed that SNNRice6mA performed better than these tools.

\section{Performance Comparison on Rice 6mA Benchmark Datasets}

To the best of our knowledge, there are six existing tools for identifying DNA 6mA sites, including i6mA-Pred (Chen et al., 2019), iDNA6mA-PseKNC (Feng et al., 2019), SDM6A
(Basith et al., 2019), iDNA6mA (Tahir et al., 2019), MM-6mAPred (Pian et al., 2019), and iDNA6mA-rice (Lv et al., 2019). Among them, i6mA-Pred, SDM6A, iDNA6mA, MM-6mAPred, and iDNA6mA-rice were all built based on the $6 \mathrm{~mA}$ sites data in rice genome, which could predict the $6 \mathrm{~mA}$ sites in rice genome. In addition, iDNA6mA-PseKNC is a tool built based on M. musculus dataset and can be applied in many other species (Feng et al., 2019). We examined whether iDNA6mA-PseKNC can predict $6 \mathrm{~mA}$ sites in rice genome. We used the rice benchmark $6 \mathrm{~mA}$-riceChen dataset to test the performance of iDNA6mA-PseKNC and found that the error rate of iDNA6mA-PseKNC prediction is relatively high $(\sim 58 \%)$. In this study, we thus just compared our method SNNRice6mA with the remaining five existing tools, including i6mA-Pred, SDM6A, iDNA6mA, MM-6mAPred, and iDNA6mA-rice. To be consistent with the evaluation metrics used in these studies, we used five metrics, including accuracy, sensitivity, specificity, MCC, and AUC.

Firstly, we compared SNNRice6mA with previous tools on the $6 \mathrm{~mA}$-rice-Chen dataset. The performance results of i6mA-Pred, SDM6A, iDNA6mA, MM-6mAPred, and iDNA6mA-rice were directly quoted from previous studies (Basith et al., 2019; Chen et al., 2019; Lv et al., 2019; Pian et al., 2019; Tahir et al., 2019). Note that the AUC value of MM-6mAPred was not calculated in the original study (Pian et al., 2019). We found that SNNRice6mA outperformed 6mA-Pred, SDM6A, iDNA6mA, MM-6mAPred, and iDNA6mA-rice in terms of sensitivity, specificity, accuracy, MCC, and AUC (Table 1).

Secondly, we compared SNNRice6mA with iDNA6mA-rice on the $6 \mathrm{~mA}$-rice-Lv dataset. During the peer reviews of our manuscript, Lv et al. proposed iDNA6mA-rice and released the 6mA-rice-Lv dataset (Lv et al., 2019). We used 5-fold crossvalidation in training SNNRice6mA on the $6 \mathrm{~mA}$-rice-Lv dataset, which is the same validation strategy as that of iDNA6mA-rice (Lv et al., 2019). The performance of iDNA6mA-rice on the $6 \mathrm{~mA}$-rice-Lv dataset was directly quoted from the original study (Lv et al., 2019). We found that SNNRice6mA outperformed iDNA6mA-rice in only one of the five evaluation metrics
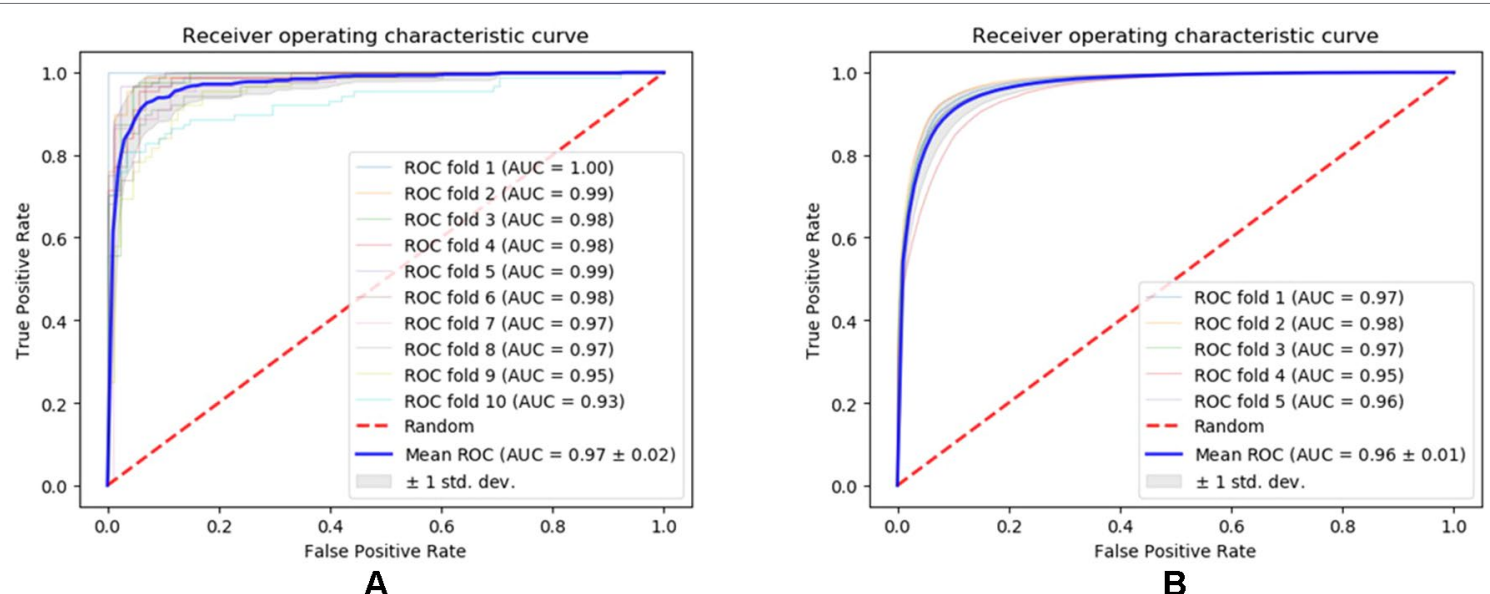

FIGURE 1 | Receiver operating characteristic curves of SNNRice6mA on testing sets of 6mA-rice-Chen dataset and 6mA-rice-Lv dataset. (A) Performance on the $6 \mathrm{~mA}$-rice-Chen dataset. (B) Performance on the 6mA-rice-Lv dataset. 
TABLE 1 | Performance comparison between SNNRice6mA and several previous methods on 6mA-Rice-Chen dataset.

\begin{tabular}{lccccc}
\hline Method & $\begin{array}{c}\text { Sensitivity } \\
(\%)\end{array}$ & $\begin{array}{c}\text { Specificity } \\
\mathbf{( \% )}\end{array}$ & $\begin{array}{c}\text { Accuracy } \\
(\%)\end{array}$ & MCC & AUC \\
\hline SNNRice6mA & 92.16 & 94.32 & 93.24 & 0.87 & 0.97 \\
SNNRice6mA & 90.34 & 92.95 & 91.65 & 0.83 & 0.98 \\
-feature & & & & & \\
i6mA-Pred & 82.95 & 83.30 & 83.13 & 0.66 & 0.89 \\
MM-6mAPred & 89.32 & 90.11 & 89.72 & 0.79 & $/$ \\
iDNA6mA & 86.70 & 86.59 & 86.64 & 0.73 & 0.93 \\
SDM6A & 85.20 & 90.90 & 88.10 & 0.76 & 0.94 \\
iDNA6mA-rice & 83.86 & 83.41 & 83.63 & 0.67 & 0.91 \\
\hline
\end{tabular}

6mA, N6-methyladenine; AUC, area under the curve; MCC, Matthews correlation coefficient.

(i.e., prediction sensitivity) (Table 2). Considering that the $6 \mathrm{~mA}$-rice-Lv dataset contains much more sample sequences than $6 \mathrm{~mA}$-rice-Chen dataset $(308,000$ vs. 1,760$)$, we sought to examine whether increasing the model complexity can improve the prediction performance of SNNRice6mA on $6 \mathrm{~mA}$-rice- $\mathrm{Lv}$ dataset. We changed the number of filters to 32 and the number of hidden units in FC layer to 64. We denoted this complex version of SNNRice6mA as SNNRice6mA-large. We retrained SNNRice6mA-large on the 6mA-rice-Lv dataset. We found that SNNRice6mA-large outperformed original SNNRice6mA in all the five evaluation metrics, and SNNRice6mA-large outperformed iDNA6mA-rice in three of the five evaluation metrics (Table 2).

TABLE 2 | Performance comparison between SNNRice6mA and iDNA6mA-Rice on 6mA-Rice-Lv dataset.

\begin{tabular}{lccccc}
\hline Method & $\begin{array}{c}\text { Sensitivity } \\
\text { (\%) }\end{array}$ & $\begin{array}{c}\text { Specificity } \\
\text { (\%) }\end{array}$ & $\begin{array}{c}\text { Accuracy } \\
\text { (\%) }\end{array}$ & MCC & AUC \\
\hline SNNRice6mA & 93.67 & 86.74 & 90.20 & 0.81 & 0.96 \\
SNNRice6mA-large & 94.33 & 89.75 & 92.04 & 0.84 & 0.97 \\
iDNA6mA-rice & 93.00 & 90.50 & 91.70 & 0.84 & 0.96 \\
\hline
\end{tabular}

6mA, N6-methyladenine; AUC, area under the curve; MCC, Matthews correlation coefficient.

\section{Comparison With Feature-Based Sequence Encoding Strategy}

To examine the effect of sequence encoding scheme, we built another model, SNNRice6mA-feature, the same as SNNRice6mA except that SNNRice6mA-feature is built based on feature-based sequence encoding. The feature-based sequence encoding we used is the same as that in a previous study (Chen et al., 2019). Two sequence features have been considered, nucleotide chemical property and nucleotide frequency. The four DNA bases, adenine (A), thymine (T), cytosine $(C)$, and guanine $(G)$, have different chemical properties. (1) $\mathrm{C}$ and $\mathrm{G}$ can from hydrogen bonds strongly, while A and T form hydrogen bonds weakly. (2) A and $\mathrm{G}$ are purines, while $\mathrm{T}$ and $\mathrm{C}$ are pyrimidines. (3) $\mathrm{A}$ and $\mathrm{C}$ are amino groups, while $\mathrm{T}$ and $\mathrm{G}$ are keto groups. We can distinguish four DNA bases in three ways, including hydrogen bond strength, base type, and amino/keto group category.
We used a triad to encode the chemical properties of four DNA bases. The first element of the triad indicates the base type; 1 means purines, and 0 means pyrimidines. The second element of the triad indicates the hydrogen bond strength; 1 means weak, while 0 means strong. The third element of the triad indicates the amino/keto group category; 1 means amino, and 0 means keto. So, we encoded A, T, C, and $\mathrm{G}$ as $(1,1,1),(0,1,0),(0,0,1)$ and $(1,0,0)$, respectively.

We used the same way as Chen et al. to calculate nucleotide frequency of every position in a sequence. The calculation formula is defined as below:

$$
d_{1}=\frac{1}{L_{1}} \sum_{j=1}^{i} f\left(N_{j}\right), f\left(N_{j}\right)=\left\{\begin{array}{l}
1, \text { if } \mathrm{N}_{j} \text { is the nucleotide concerned } \\
0, \text { otherwise }
\end{array}\right.
$$

where $d_{i}$ is the nucleotide frequency of position $i$ in a DNA sequence. $L_{i}$ is the length of the subsequence from the first position to the position $i$ of the sequence. $N_{i}$ stands for the base in position $i$ of a DNA sequence (i.e., one of the A, T, C, and G).

Combining the nucleotide chemical properties and nucleotide frequency together, each DNA sequence can be represented as a matrix, with 41 columns and 4 rows. Each column of the matrix represents a specific DNA base. For each column, the first three elements represent the nucleotide chemical properties, and the last element represents its nucleotide frequency.

We trained SNNRice6mA-feature on the $6 \mathrm{~mA}$-riceChen dataset by using the feature-based sequence encoding method above. SNNRice6mA-feature was trained and tested in the same way as SNNRice6mA. SNNRice6mA-feature outperformed SNNRice6mA in only one of the five evaluation metrics (Table 1).

\section{Cross-Species Evaluation}

We next tested whether model trained on rice datasets can be used to predict DNA $6 \mathrm{~mA}$ sites in other species. We used the M. musculus $6 \mathrm{~mA}$ dataset proposed in a previous study (Feng et al., 2019) and denoted this dataset as $6 \mathrm{~mA}$-mouse-Feng. $6 \mathrm{~mA}$-mouse-Feng dataset contains $1,9346 \mathrm{~mA}$ site containing sequences and 1,934 non- $6 \mathrm{~mA}$ site containing sequences. We used this independent dataset as test data. We evaluated the performance of SNNRice6mA, which was trained on rice 6mA-rice-Lv dataset, on the M. musculus test data. We also performed similar evaluation for three of the five rice $6 \mathrm{~mA}$ prediction methods, including i6mA-Pred (Chen et al., 2019), iDNA6mA (Tahir et al., 2019), and MM-6mAPred (Pian et al., 2019). For the remaining two rice $6 \mathrm{~mA}$ prediction methods, SDM6A (Basith et al., 2019) and iDNA6mA-rice (Lv et al., 2019), we encountered errors when using these two tools (Figures S3, S4). We thus excluded these two methods for evaluation. We found that SNNRice6mA achieved predicted accuracy of $61.81 \%$, which was higher than those of the other three methods (52.43\% for i6mA-Pred, $41.93 \%$ for iDNA6mA, $44.11 \%$ for MM-6mAPred). 


\section{CONCLUSIONS}

In this study, we proposed a deep learning model SNNRice6mA to identify DNA $6 \mathrm{~mA}$ sites in rice genome. SNNRice6mA got similar predicted accuracies on the two rice datasets $(\sim 93 \%$ and $\sim 92 \%$ ). SNNRice6mA performed better than previous methods in prediction of $6 \mathrm{~mA}$ sites. Though the limited size of available training dataset might bias the generalization of the model, we used some techniques (e.g., regularization) to minimize this artifact. We expect that our method can facilitate the analysis of DNA $6 \mathrm{~mA}$ sites in the rice genome. However, there are some limitations for our method. First, the feature ranking is not possible in the current version. Second, there is room for improvement on the performance of rice data-trained SNNRice6mA on M. musculus dataset.

\section{DATA AVAILABILITY STATEMENT}

Publicly available datasets were analyzed in this study. These data can be found here: https://github.com/yuht4/SNNRice6mA.

\section{REFERENCES}

Au, KG., Welsh, K., and Modrich, P. (1992). Initiation of methyl-directed mismatch repair. J. Biol. Chem. 267, 12142-12148.

Basith, S., Manavalan, B., Shin, T. H., and Lee, G. (2019). SDM6A: A web-based integrative machine-learning framework for predicting $6 \mathrm{~mA}$ sites in the rice genome. Mol. Ther. Nucleic Acids. 18, 131-141. doi: 10.1016/j.omtn.2019.08.011

Boughorbel, S., Jarray, F., and El-Anbari, M. (2017). Optimal classifier for imbalanced data using Matthews correlation coefficient metric. PLoS One 12, e0177678. doi: 10.1371/journal.pone.0177678

Campbell, J. L., and Kleckner, N. (1990). E. coli oriC and the dnaA gene promoter are sequestered from dam methyltransferase following the passage of the chromosomal replication fork. Cell 62pp, 967-979. doi: 10.1016/0092-8674(90)90271-F

Chen, W., Lv, H., Nie, F., and Lin, H. (2019). i6mA-Pred: identifying DNA N6-methyladenine sites in the rice genome. Bioinformatics. 35, 2796-2800. doi: 10.1093/bioinformatics/btz015

Feng, P., Yang, H., Ding, H., Lin, H., Chen, W., and Chou, K.-C. (2019). iDNA6mAPseKNC: identifying DNA N6-methyladenosine sites by incorporating nucleotide physicochemical properties into PseKNC. Genomics 111, 96-102. doi: 10.1016/j.ygeno.2018.01.005

Flusberg, B. A., Webster, D. R., Lee, J. H., Travers, K. J., Olivares, E. C., Clark, T. A., et al. (2010). Direct detection of DNA methylation during singlemolecule, real-time sequencing. Nat. Methods 7, 461-465. doi: 10.1038/ nmeth. 1459

Greer, E. L., Blanco, M. A., Gu, L., Sendinc, E., Liu, J., Aristizábal-Corrales, D., et al. (2015). DNA Methylation on N6-Adenine in C. elegans. Cell 161, 868-878. doi: 10.1016/j.cell.2015.04.005

Koziol, M. J., Bradshaw, C. R., Allen, G. E., Costa, A. S. H., and Frezza, C. (2016). Identification of methylated deoxyadenosines in genomic DNA by dA(6m) DNA Immunoprecipitation. Bio-protocol 6, e1990. doi: 10.21769/ BioProtoc. 1990

Krais, A. M., Cornelius, M. G., and Schmeiser, H. H. (2010). Genomic N(6)methyladenine determination by MEKC with LIF. Electrophoresis 31, 3548-3551. doi: 10.1002/elps.201000357

Low, D. A., Weyand, N. J., and Mahan, M. J. (2001). Roles of DNA adenine methylation in regulating bacterial gene expression and virulence. Infect. Immun. 69, 7197-7204. doi: 10.1128/IAI.69.12.7197-7204.2001

Lv, H., Dao, F.-Y., Guan, Z.-X., Zhang, D., Tan, J.-X., Zhang, Y., et al. (2019). iDNA6mA-Rice: a computational tool for detecting N6-methyladenine sites in rice. Front. Genet. 10, 1-11. doi: 10.3389/fgene.2019.00793

\section{AUTHOR CONTRIBUTIONS}

HY and ZD designed the study, analyzed the results, and drafted the manuscript. HY implemented the algorithms and carried out the experiments.

\section{FUNDING}

This work was supported by National Natural Science Foundation of China (NSFC) (grant 61872395, U1611265), by Natural Science Foundation of Guangdong Province (2018A030313285), and also by Pearl River Nova Program of Guangzhou (201710010044).

\section{SUPPLEMENTARY MATERIAL}

The Supplementary Material for this article can be found online at: https://www.frontiersin.org/articles/10.3389/fgene.2019.01071/ full\#supplementary-material

Matthews, B. W. (1975). Comparison of the predicted and observed secondary structure of T4 phage lysozyme. Biochim. et Biophysi. Acta (BBA) - Protein Struct. 405, 442-451. doi: 10.1016/0005-2795(75)90109-9

O'brown, Z. K., and Greer, E. L. (2016). "N6-Methyladenine: A Conserved and Dynamic DNA Mark," in DNA Methyltransferases - Role and Function. Eds. A. Jeltsch and R. Z. Jurkowska (Cham: Springer International Publishing), 213-246. doi: 10.1007/978-3-319-43624-1_10

Pian, C., Zhang, G., Li, F., and Fan, X. (2019). MM-6mAPred: identifying DNA N6-methyladenine sites based on Markov model. Bioinformatics. doi: 10.1093/ bioinformatics/btz556

Pomraning, K. R., Smith, K. M., and Freitag, M. (2009). Genome-wide high throughput analysis of DNA methylation in eukaryotes. Methods 47, 142-150. doi: 10.1016/j.ymeth.2008.09.022

Robbins-Manke, J. L., Zdraveski, Z. Z., Marinus, M., and Essigmann, J. M. (2005). Analysis of global gene expression and double-strand-break formation in DNA adenine methyltransferase- and mismatch repair-deficient Escherichia coli. J. Bacterial. 187, 7027-7037. doi: 10.1128/JB.187.20.7027-7037.2005

Tahir, M., Tayara, H., and Chong, K. T. (2019). iDNA6mA (5-step rule): identification of DNA N6-methyladenine sites in the rice genome by intelligent computational model via Chou's 5-step rule. Chemometr. Intell. Lab. Syst. 189, 96-101. doi: 10.1016/j.chemolab.2019.04.007

Wu, Y., and He, K. (2018). "Group normalization", in Computer Vision - ECCV 2018. LNCS, vol. 11217. Eds. V. Ferrari, M. Hebert, C. Sminchisescu and Y. Weiss. 3-19. doi: 10.1007/978-3-030-01261-8_1

Zhang, Z., Zhao, Y., Liao, X., Shi, W., Li, K., Zou, Q., et al. (2018). Deep learning in omics: a survey and guideline. Brief Funct. Genomic 18, 41-57. doi: 10.1093/ bfgp/ely030

Zou, Q., Xing, P., Wei, L., and Liu, B. (2019). Gene2vec: gene subsequence embedding for prediction of mammalian N6-methyladenosine sites from mRNA. RNA 25, 205-218. doi: 10.1261/rna.069112.118

Conflict of Interest: The authors declare that the research was conducted in the absence of any commercial or financial relationships that could be construed as a potential conflict of interest.

Copyright $\odot 2019$ Yu and Dai. This is an open-access article distributed under the terms of the Creative Commons Attribution License (CC BY). The use, distribution or reproduction in other forums is permitted, provided the original author $(s)$ and the copyright owner(s) are credited and that the original publication in this journal is cited, in accordance with accepted academic practice. No use, distribution or reproduction is permitted which does not comply with these terms. 